\title{
Regiochemistry-driven Organic Electrochemical Transistor Performance Enhancement in Ethylene Glycol Functionalized Polythiophenes
}

\author{
Rawad K. Hallani, ${ }^{1}$ Bryan D. Paulsen, ${ }^{2}$ Anthony J. Petty II, ${ }^{2}$ Rajendar Sheelamanthula, ${ }^{1}$ \\ Maximilian Moser, ${ }^{6}$ Karl J. Thorley, ${ }^{4}$ Wonil Sohn, ${ }^{2}$ Reem B. Rashid, ${ }^{2}$ Achilleas Savva, ${ }^{5}$ Stefania \\ Moro, ${ }^{7}$ Joseph P. Parker, ${ }^{7}$ Oscar Drury, ${ }^{7}$ Maryam Alsufyani, ${ }^{1}$ Marios Neophytou, ${ }^{1}$ Jan Kosco $^{1}$, \\ Sahika Inal, ${ }^{5}$ Giovanni Costantini, ${ }^{7}$ Jonathan Rivnay, ${ }^{2,3}$ and Iain McCulloch. ${ }^{1,6 *}$ \\ ${ }^{1}$ King Abdullah University of Science and Technology (KAUST), Physical Science and Engineering Division, \\ Thuwal, 23955-6900, Saudi Arabia. Rawad.hallani@kaust.edu.sa, rajendar.sheelamanthula@gmail.com, \\ maryam.alsufyani@kaust.edu.sa, $\quad$ marios.neophytou@kaust.edu.sa, an.kosco@kaust.edu.sa, \\ iain.mcculloch@kaust.edu.sa.
}

${ }^{2}$ Northwestern University, Department of Biomedical Engineering, 2145 Sheridan Rd, Evanston, IL 60208, USA. $\quad$ bryan.paulsen@northwestern.edu, $\quad$ wonilsohn2021@u.northwestern.edu, reemrashid2022@u.northwestern.edu, jrivnay@northwestern.edu.

${ }^{3}$ Simpson Querrey Institute, Northwestern University, Chicago, Illinois 60611, USA. jrivnay@northwestern.edu.

${ }^{4}$ Department of Chemistry, University of Kentucky, Lexington, Kentucky 40506, USA. kjth227@g.uky.edu.

${ }^{5}$ King Abdullah University of Science and Technology (KAUST), Organic Bioelectronics Laboratory, Biological and Environmental Science and Engineering, Thuwal, 23955-6900, Saudi Arabia. as3024@cam.ac.uk, sahika.inal@kaust.edu.sa.

${ }^{6}$ Department of Chemistry, Chemistry Research Laboratory, University of Oxford, Oxford, OX1 3TA, UK. iain.mcculloch@chem.ox.ac.uk.

${ }^{7}$ Department of Chemistry, University of Warwick, Coventry CV4 7AL, UK. Stefania.Moro@warwick.ac.uk, Joseph.Parker@warwick.ac.uk, O.Drury@warwick.ac.uk, G.Costantini@warwick.ac.uk. 


\section{Table of Contents}

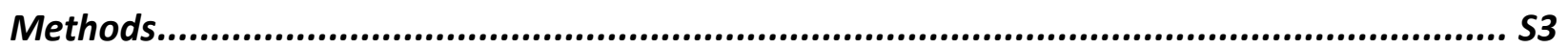

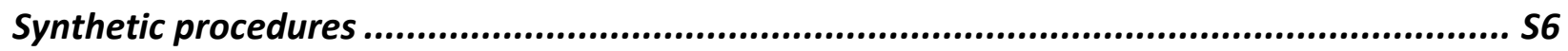

DFT calculations of the dihedral angles ..................................................................S11

Thermal analysis .....................................................................................S14

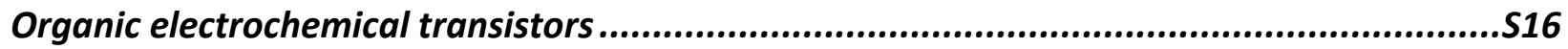

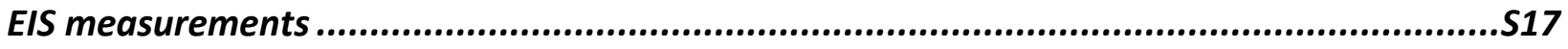

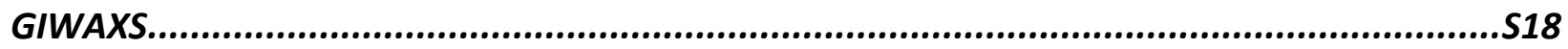

Scanning Tunneling Microscopy.......................................................................S19

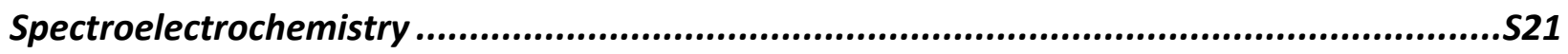

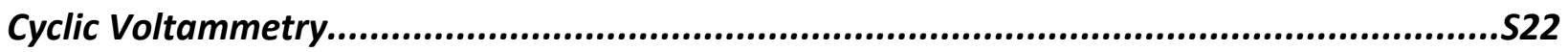

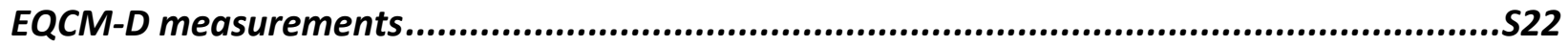

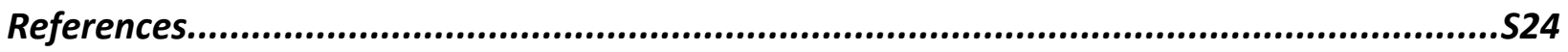




\section{General Methods}

Materials characterization. All chemicals were purchased from Sigma Aldrich and used as received. ${ }^{1} \mathrm{H}$ and ${ }^{13} \mathrm{C}$ NMR spectra were acquired on either a Bruker Avance III $400 \mathrm{MHz}$ NMR spectrometer equipped with a BBFO SmartProbe or a Bruker Avance III $500 \mathrm{MHz}$ NMR spectrometer equipped with a BBFO CryoProbe. Gas chromatography-mass spectrometry (GC-MS) was carried out on an Agilent Technologies 7693. Gel permeation chromatography (GPC) was measured using a PLgel 10 um Mixed-B column on an Agilent Technologies 1260 Infinity system with DMF as the mobile phase at $80{ }^{\circ} \mathrm{C}$. Thermogravimetric analysis (TGA) and differential scanning calorimetry (DSC) were performed using a Mettler Toledo TGA/DSC 1LF/UMX and employing $70 \mu \mathrm{L}$ ceramic crucibles into which the samples were placed. Samples were heated under a nitrogen purge gas flow rate of $50 \mathrm{~mL} \mathrm{~min}^{-1}$ and a rate of $10{ }^{\circ} \mathrm{C} \mathrm{min}^{-1}$. Photoelectron spectroscopy in air (PESA): These measurements were conducted at room temperature using a Riken Instruments AC-2 model to determine the ionization potential of the polymers.

Computational details. All calculations were carried out using Gaussian 16 Rev A.02 software. ${ }^{1}$ Torsional potentials were determined on polymers of 4 repeating TT and $2 \mathrm{~T}$ units, varying the centermost TT-T or T-T bonds. Torsional potentials were calculated using B3LYP-D3/6-31G* as the choice of range separation parameter for the $\omega \mathrm{B} 97 \mathrm{XD}$ functional may not be the same at each dihedral angle. Full geometry optimization and electronic properties of the polymers were investigated using an 'IP-tuned' range separated $\omega \mathrm{B} 97 \mathrm{XD}$ and $6-31 \mathrm{G}^{*}$ basis set to give an improved description of electronic structure over standard hybrid functionals. ${ }^{2}$ Charge distribution 
was visualized by dividing the polymer into fragments of either TT or 2T, and using the Gaussian (fragment=n) designation in the input files. Hirshfeld charge analysis was performed, with the output file directly reporting charge per fragment. The non-zero fragment charges of the neutral polymer were subtracted from the charges of the radical cation polymer to give the polaron distribution.

Electrochemical analysis. Cyclic voltammetry (CV) was conducted with an Autolab PGSTAT101 featuring a standard three-electrode configuration. A polymer coated ITO glass slide, a platinum wire and an $\mathrm{Ag} / \mathrm{AgCl}$ wire were used as the working, counter and reference electrodes respectively. Polymer coated ITO glass slide samples were prepared by freshly cutting ITO coated glass substrates, which were subsequently cleaned by sonication in soapy water, water, acetone and isopropanol. Polymers were deposited by spin coating $50 \mu \mathrm{L}$ of $5 \mathrm{mg} \mathrm{mL}^{-1}$ polymer solutions onto the freshly cleaned substrates. CV measurements were conducted using either an organic electrolyte, consisting of a $0.1 \mathrm{M}$ tetrabutylammonium hexafluorophosphate in acetonitrile solution, or an aqueous one, consisting of a $0.1 \mathrm{M}$ sodium chloride in distilled water solution. Cyclic voltammetry determined ionization potentials (IPs) were determined through the following equation: $I P(\mathrm{eV})=\left(E_{o x}-E_{F c}+5.1\right)$, where $\mathrm{E}_{\mathrm{ox}}$ is the onset of polymer oxidation employing a $0.1 \mathrm{M}$ solution of tetrabutylammonium hexafluorophosphate in acetonitrile as the supporting electrolyte and $\mathrm{E}_{\mathrm{Fc}}$ is the half-wave potential of the ferrocene/ferrocenium $\left(\mathrm{Fc} / \mathrm{Fc}^{+}\right)$redox couple.

Grazing Incidence Wide Angle X-ray Scattering. 2-D GIWAXS films were spun from polymer solutions ( $5 \mathrm{mg} \mathrm{mL}^{-1}$ in chloroform) on polished Si wafer substrates (University Wafer). Scattering was carried out at the Advanced Photon Source at Argonne National Laboratory on beam line 8ID-E at room temperature under vacuum with $10.92 \mathrm{keV}(\lambda=1.135 \AA)$ synchrotron radiation, with a $0.14^{\circ}$ incident angle, and measured with a Pilatus $1 \mathrm{M}$ hybrid pixel array detector during 10 second exposures. Data analysis was carried out with GIXSGUI Matlab toolbox ${ }^{3}$ and with custom curve fitting software.

Scanning Tunneling Microscopy. Measurements were performed with a low temperature (LT) scanning tunneling microscope (STM) operated in ultrahigh vacuum (UHV) condition and equipped with an electrospray deposition (ESD) setup. Both p(g2T-TT) and pgBTTT samples were dissolved in chloroform at a concentration of $\approx 0.025 \mathrm{~g} / \mathrm{L}$. The molecules were deposited from solution by a 4-stage ESD system (Molecularspray Ltd.). The deposition current was monitored 
on the target substrate and typical deposition charges were $8 \mathrm{pAh}$. Au(111) on mica thin films (Georg Albert PVD, $300 \mathrm{~nm}$ thickness) were used as substrates and were cleaned in UHV by cycles of argon sputtering $(1 \mathrm{kV})$ and annealing to $500{ }^{\circ} \mathrm{C}$. The LT-STM (CreaTec Fischer \& Co. GmbH) was kept at $-196{ }^{\circ} \mathrm{C}$ by a liquid nitrogen bath cryostat. All images were acquired in constant current feedback mode with an electrochemically etched tungsten tip that was treated by argon sputtering after insertion in UHV, in order to remove the oxide. The STM images were processed with $\mathrm{WSxM},{ }^{4}$ the molecular models were made and optimized in the Avogadro molecular editor, ${ }^{5}$ and their comparison with and superposition on the STM images was performed with the LMAPper software. ${ }^{6}$

Optical spectroscopy. Spectroelectrochemistry was conducted by placing the electrochemical setup employed for CV within the UV-1800 Shimadzu UV-Vis spectrometer previously employed for determining the optical properties of the polymers. Samples for solid state spectroelectrochemical evaluation were prepared analogously to those employed for CV.

Organic Electrochemical Transistors. OECTs test chips were prepared following microfabrication techniques previously reported. ${ }^{7}$ OECT channels were fabricated by drop casting from chloroform ( $5 \mathrm{mg} \mathrm{mL}^{-1}$ ) onto OECT test chips at room temperature, followed by patterning via peeling a sacrificial parylene layer, and a rinse in deionized water. OECTs were gated with aqueous $100 \mathrm{mM} \mathrm{NaCl}$ using a $\mathrm{Ag} / \mathrm{AgCl}$ pellet as the faradaic gate electrode. ${ }^{8}$ Electrical characterization (output and transfer curves) of the OECTs were carried out using NI sourcemeasure units controlled by custom LabView code. Capacitance was measured via electrochemical impedance spectroscopy using a Metrohm potentiostat with frequency response analyzer.

\section{Electrochemical quartz crystal microbalance with dissipation of energy (eQCM-D)}

measurements. eQCM-D measurements were performed using a Q-sense analyzer (QE401, Biolin Scientific). Swelling measurements were performed as follows. First, the QCM-D response of the bare $\mathrm{Au}$ sensors was recorded in air, followed by injection of $\mathrm{NaCl}$ (aq.) $0.1 \mathrm{M}$ solution into the chamber. This resulted in significant shifts in $f$ and $D$, due to the density differences between the two media, which must be excluded from the swelling percentage calculation. The measurements were then stopped, the sensors were removed, and the polymer layers were cast directly on the same sensors from $4 \mathrm{mg} \mathrm{mL}^{-1}$ solutions in chloroform at $1000 \mathrm{rpm}$. The 
absolute $f$ value for each polymer-coated sensor was obtained both in air and in $\mathrm{NaCl}$ (aq.) $0.1 \mathrm{M}$ after the $f$ signal was perfectly flat (i.e., $\mathrm{D} f<0.5 \mathrm{~Hz} / 2 \mathrm{~min}$ ), assuring that the system was in equilibrium. Then, the absolute difference in $f$ for multiple overtones between the bare sensor and the polymer-coated sensors was compared, both in air and in the electrolyte, using the function "stitched data" of Q-soft software. This function compares the selected datasets based on the raw frequencies measured and excludes the effect of the different densities between the two media (Figure S14). Thus, the difference of the $f$ values of the stitched data is directly analogous to the mass absorbed by the polymer $(m)$ in both media, which is calculated by using the Sauerbrey equation: $\Delta m=\frac{-17.7}{\mathrm{n}} \Delta f_{\mathrm{n}}$. For eQCM-D measurements, the Q-sense electrochemistry module, comprising three-electrode setup, was coupled with an Autolab PGstat128N potentiostat. Since the films become soft and uptake a significant amount of water under doping potential, the KelvinVoigt viscoelastic model was used to fit the data and quantify the mass, as described in detail elsewhere. ${ }^{9}$

\section{Synthetic procedures}

$\mathrm{p}(\mathrm{g} 2 \mathrm{~T}-\mathrm{TT})$ was synthesized following a literature procedure. ${ }^{10}$

\section{Synthesis of the monomers and polymers.}
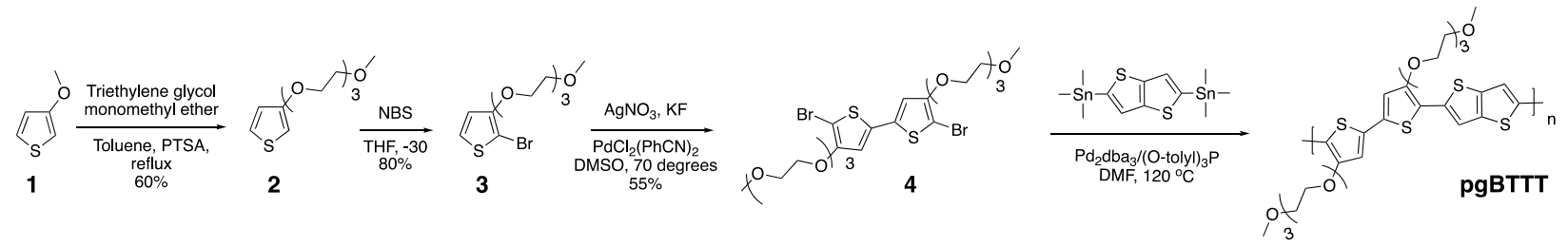

3-(2-methoxyethoxy)thiophene (2): A $250 \mathrm{~mL}$ RBF was charged with triethylene glycol monomethyl ether (8.63 g, $53.0 \mathrm{mmol}, 1.50$ eq.), 1 (4 g, $35 \mathrm{mmol}, 1$ eq) and PTSA (667 mg, 3.5 mmol, 1 eq). The reactants were dissolved in $150 \mathrm{~mL}$ of toluene and the reaction was refluxed overnight. The consumption of the starting material was monitored via GC-MS. Toluene was evaporated and the crude product was purified using silica flash column chromatography employing a 4:1 v/v mixture of hexane: DCM followed by a 5:2 v/v mixture of hexane:ethyl acetate 
as the eluents to obtain 2 as a pale yellow viscous oil $(5.17 \mathrm{~g}, 21 \mathrm{mmol}, 60 \%$ yield). Experimental measurements and characterizations are in agreement with the data reported in literature. ${ }^{10}{ }^{1} \mathrm{H}$ NMR (400 MHz, CDCl3): $\delta 7.14$ (dd, 1H, J = 5.1, $3 \mathrm{~Hz}$ ), 6.74 (dd, 1H, J = 5, $1.7 \mathrm{~Hz}), 6.25$ (dd, $1 \mathrm{H}, \mathrm{J}=3.1,1.6 \mathrm{~Hz}), 4.11(\mathrm{t}, 2 \mathrm{H}, \mathrm{J}=4.8 \mathrm{~Hz}), 3.84(\mathrm{t}, 2 \mathrm{H}, \mathrm{J}=4.7 \mathrm{~Hz}), 3.72(\mathrm{dd}, 2 \mathrm{H}, \mathrm{J}=6.3,3.5$ Hz), 3.70 (m, 4H), 3.52 (dd, 2H, J = 5.3, 3.6 Hz), 3.38 (s, 3H). MS (EI): m/z 246 [M+].

2-bromo-3-(2-methoxyethoxy)thiophene (3): In a flame dried RBF 2 (3.2g, 13mmol, 1eq) was dissolved in $60 \mathrm{ml}$ THF and the RBF placed in a dry ice bath where the temperature was kept at $30{ }^{\circ} \mathrm{C}$. After $15 \mathrm{~min}$ of cooling, $\mathrm{n}$-bromosuccinimide $(2.32 \mathrm{~g}, 13 \mathrm{mmol}$, 1eq) was added in one portion in the dark. The consumption of the starting materials was monitored using GC-MS. Upon completion, the reaction was quenched with water, extracted with diethyl ether, dried over $\mathrm{MgSO}_{4}$, and concentrated under reduced pressure. The crude product was purified using silica flash column chromatography employing a 6:4 v/v mixture of ethyl acetate:hexane as the eluent, to yield $\mathbf{3}$ as a yellow oil (3.28 g, $10.1 \mathrm{mmol}, 78 \%$ yield). Experimental measurements and characterizations are in agreement with the data reported in literature. ${ }^{11}{ }^{1} \mathrm{H} \mathrm{NMR}(400 \mathrm{MHz}, \mathrm{CDCl} 3): \delta(\mathrm{ppm}) 7.17$ (d, $1 \mathrm{H}, \mathrm{J}=5.9 \mathrm{~Hz}), 6.76(\mathrm{~d}, 1 \mathrm{H}, \mathrm{J}=6.0 \mathrm{~Hz}), 4.16(\mathrm{t}, 2 \mathrm{H}, \mathrm{J}=4.0 \mathrm{~Hz}), 3.80(\mathrm{t}, 2 \mathrm{H}, \mathrm{J}=4.0 \mathrm{~Hz}), 3.71$ (m, 2H), $3.68-3.60(\mathrm{~m}, 4 \mathrm{H}), 3.53(\mathrm{~m}, 2 \mathrm{H}), 3.36$ (s, 3H). MS (EI): m/z 324 [M+].

5,5'-dibromo-4,4'-bis(2-methoxyethoxy)-2,2'-bithiophene (4): $10 \mathrm{~mL}$ of anhydrous DMSO was added to a dry pressure vial and purged with nitrogen for 20 minutes. 3 was added $(1 \mathrm{~g}, 3.067$ mmol, 1 eq.) followed by silver nitrate $(1.042,6.134 \mathrm{mmol}, 2 \mathrm{eq}$.), potassium fluoride $(356 \mathrm{mg}$, $6.134 \mathrm{mmol} 2 \mathrm{eq}$.), and bis(benzonitrile)palladium dichloride $\left(\mathrm{PdCl}_{2}(\mathrm{PhCN})_{2}\right)(35 \mathrm{mg}, 0.092$ mmol, 0.03 eq.) The vial was heated up to $90{ }^{\circ} \mathrm{C}$ and left to react for $3 \mathrm{~h}$. The reaction was monitored by GCMS, which showed the consumption of the starting material. The reaction was cooled down to room temperature and quenched with water. The aqueous layer was extracted with diethyl ether, the combined organic layers washed with water and brine before being dried over $\mathrm{MgSO}_{4}$. Excess solvent was removed under reduced pressure. The crude product was purified using silica flash column chromatography employing ethyl acetate as the eluent to yield $\mathbf{4}$ as a white solid (0.55 g, $0.85 \mathrm{mmol}, 55 \%$ yield). ${ }^{1} \mathrm{H}$ NMR (400 MHz, CDCl3): $\delta$ (ppm) 6.81 (s, 2H), $4.24(\mathrm{t}, 4 \mathrm{H}, \mathrm{J}=4.8 \mathrm{~Hz}), 3.85(\mathrm{t}, 4 \mathrm{H}, \mathrm{J}=4.8 \mathrm{~Hz}), 3.77$ (dd, 4H, J = 5.9, 3.6 Hz), 3.74-3.63 (m, 8H), $3.57(\mathrm{dd}, 4 \mathrm{H}, \mathrm{J}=5.7,3.7), 3.39(\mathrm{~s}, 6 \mathrm{H}) .{ }^{13} \mathrm{C} \mathrm{NMR}(100 \mathrm{MHz}, \mathrm{CDCl} 3): \delta(\mathrm{ppm}) 154.4,134.8$, 
114.0, 91.7, 71.9, 71.0, 70.7, 70.6, 69.8, 59.0. HRMS (ESI-TOF) m/z: calculated 648.99576, found 648.99457.

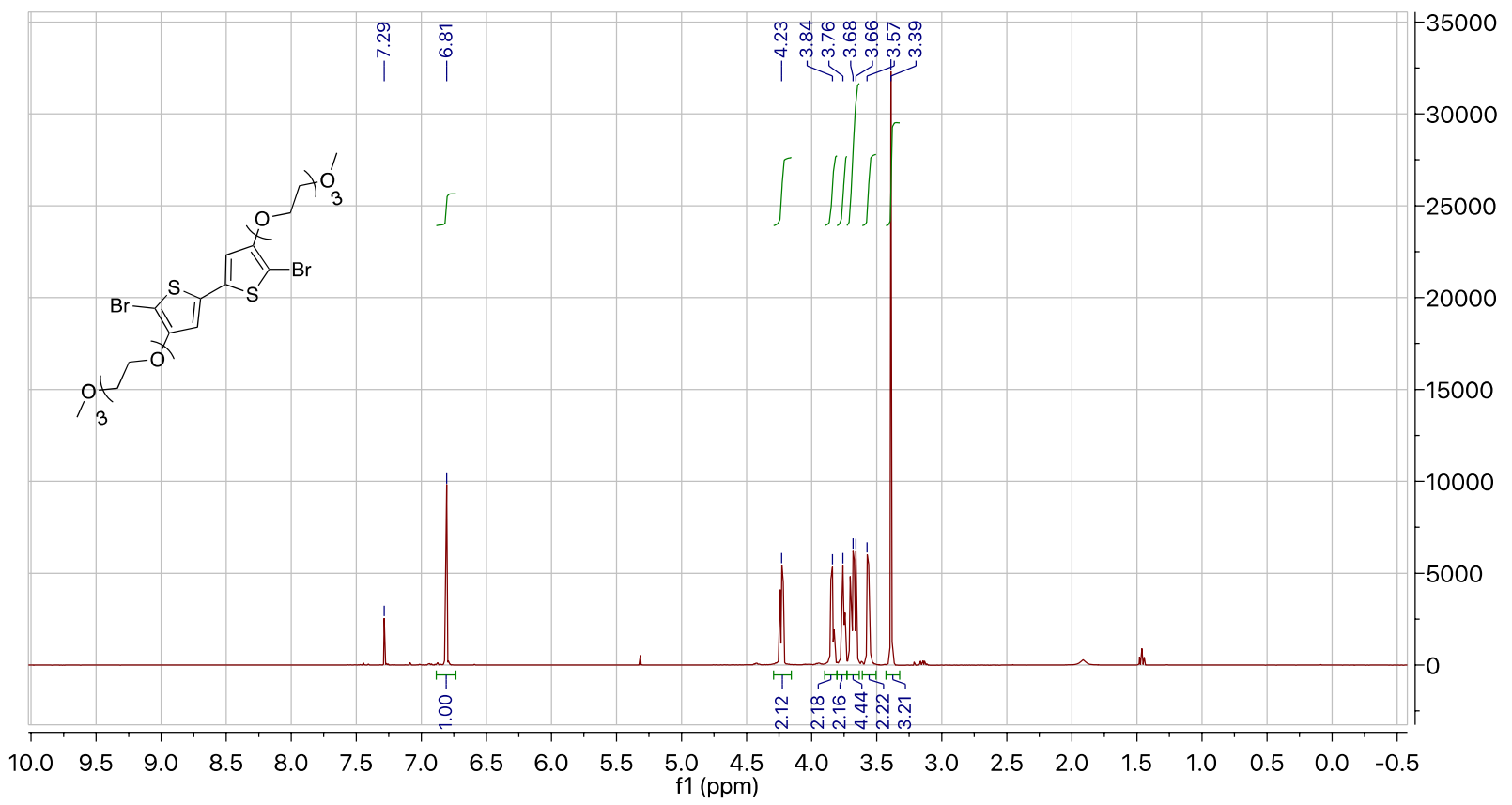

Figure S1. ${ }^{1} \mathrm{H}$ NMR of 5,5'-dibromo-4,4'-bis(2-methoxyethoxy)-2,2'-bithiophene (4). 


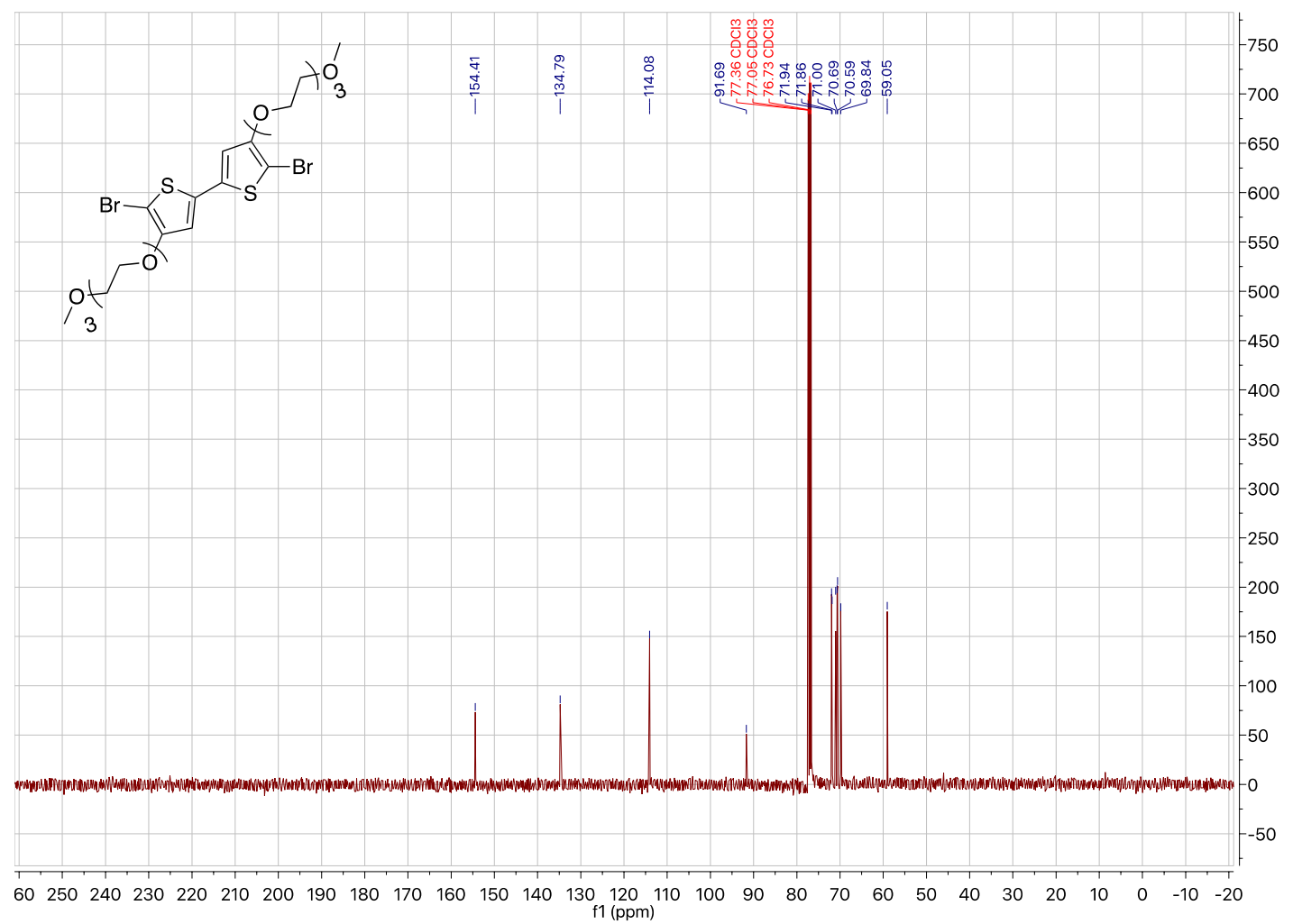

Figure S2. ${ }^{13} \mathrm{C}$ NMR 5,5'-dibromo-4,4'-bis(2-methoxyethoxy)-2,2'-bithiophene (4).

pgBTTT: 5,5'-dibromo-4,4'-bis(2-(2-(2-methoxyethoxy)ethoxy)ethoxy)-2,2'-bithiophene (100 $\mathrm{mg}, 0.15 \mathrm{mmol}, 1$ eq.), and 2,5-bis(trimethylstannyl)thieno[3,2-b]thiophene (71.73 $\mathrm{mg}, 0.15$ mmol, 1 eq.) were added to a dry microwave vial and dissolved in $3.0 \mathrm{~mL}$ of degassed anhydrous DMF. The reaction mixture was subsequently purged with argon for another $20 \mathrm{~min} . \mathrm{Pd}_{2}(\mathrm{dba})_{3}$ (2.8 mg, $0.003 \mathrm{mmol}, 0.02$ eq.) and $\mathrm{P}(o \text {-tol })_{3}(3.74 \mathrm{mg}, 0.012 \mathrm{mmol}, 0.08$ eq.) were added, the reaction mixture purged for 5 additional minutes before being heated to $100{ }^{\circ} \mathrm{C}$. The reaction formed a blue gel during the polymerization. After heating for 9 hours, the end-capping procedure was carried out.

End capping procedure: $0.2 \mathrm{~mL}$ of degassed $\mathrm{DMF}$ was added followed by $\mathrm{Pd}_{2}(\mathrm{dba})_{3}$ and $\mathrm{P}(\mathrm{o}-\mathrm{tolyl})_{3}$ $\left(1: 8, \mathrm{Pd}_{2}(\mathrm{dba})_{3}: \mathrm{P}(\mathrm{o} \text {-tolyl })_{3}\right.$ molar ratio; Pd loading 0.0067 equiv.). 2-(Tributylstannyl)thiophene (0.2 equiv) was added, and the reaction was left to stir at $120{ }^{\circ} \mathrm{C}$ for $15 \mathrm{~min}$. Finally, 2bromothiophene ( 0.4 equiv) was added, and the reaction was left to stir at $120^{\circ} \mathrm{C}$ for $15 \mathrm{~min}$. 
The reaction mixture was then cooled to room temperature, and the blue polymer was collected in a thimble. Soxhlet extractions were carried out with methanol (16 h), acetone (16 h), hexane (16 h), THF (16 h) and chloroform (16 h), with the polymer eluting in the chloroform fraction. Excess solvent was removed under reduced pressure. The polymer was dissolved once again in chloroform and precipitated into methanol. Finally, the polymer collected by suction filtration and dried under high vacuum before being stored under argon atmosphere. pgBTTT was obtained as a blue colored solid (23 mg, 24\% yield). GPC (DMF, $\left.45^{\circ} \mathrm{C}\right) \mathrm{M}_{\mathrm{n}}=10 \mathrm{kDa}, \mathrm{M}_{\mathrm{w}}=24 \mathrm{kDa} ;{ }^{1} \mathrm{H} \mathrm{NMR}(400 \mathrm{MHz}$, $\mathrm{CDCl} 3$ ): $\delta$ (ppm) 6.99 (br s), 4.17 (br s), 3.68 (br m), 3.58(br s), 3.39 (br s).

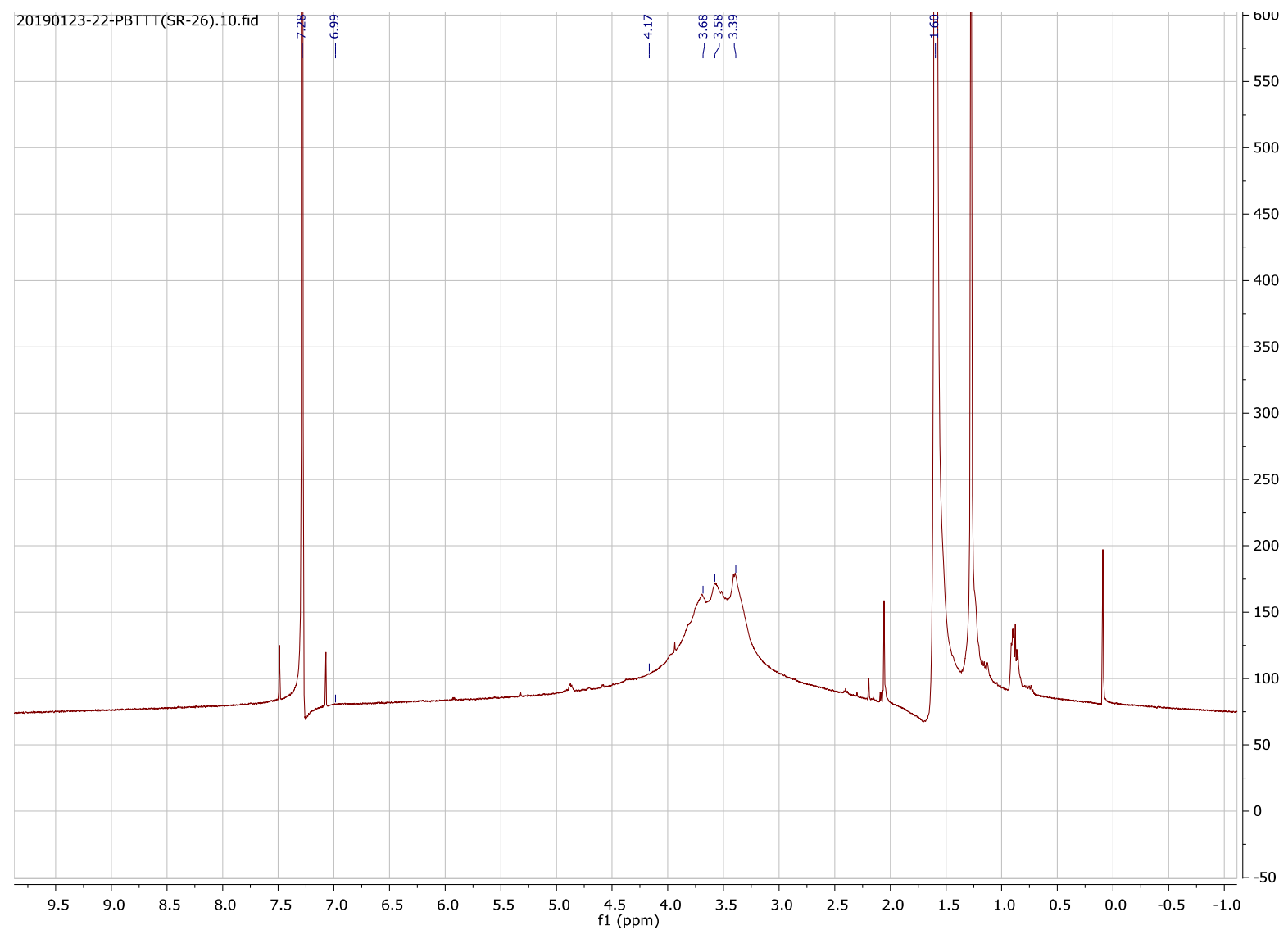

Figure S3. ${ }^{1} \mathrm{H}$ NMR of pgBTTT (5) 


\section{DFT calculations of the dihedral angles}

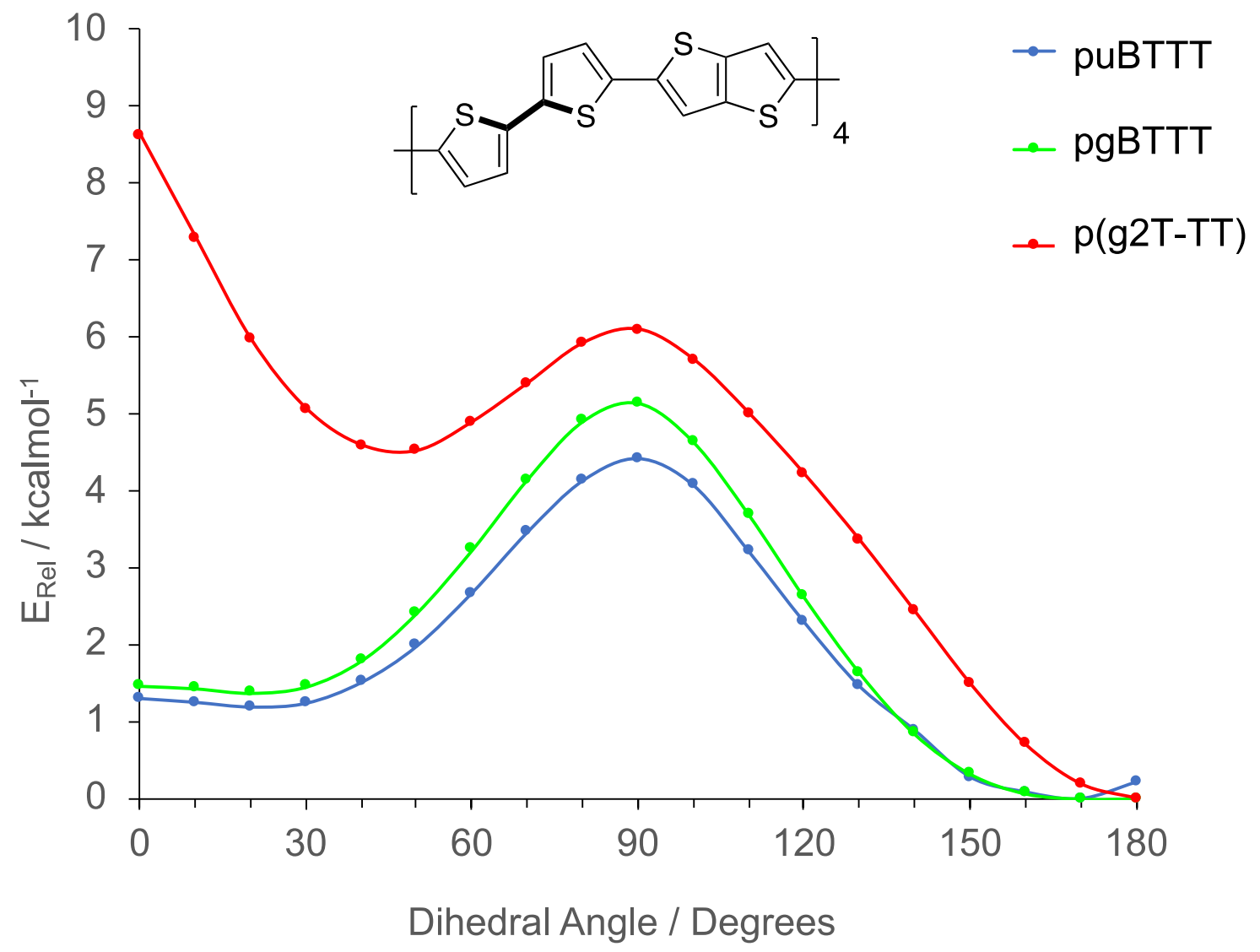

Figure S4. Torsional potential energy surface for puBTTT (blue), pgBTTT (green) and pg2T-TT (red) polymers about the centermost T-T bond of model oligomers with 4 repeat units, calculated using B3LYP$\mathrm{D} 3 / 6-31 \mathrm{G}^{*}$. The rotated bond is highlighted as bold, and the $180^{\circ}$ conformer is shown. 


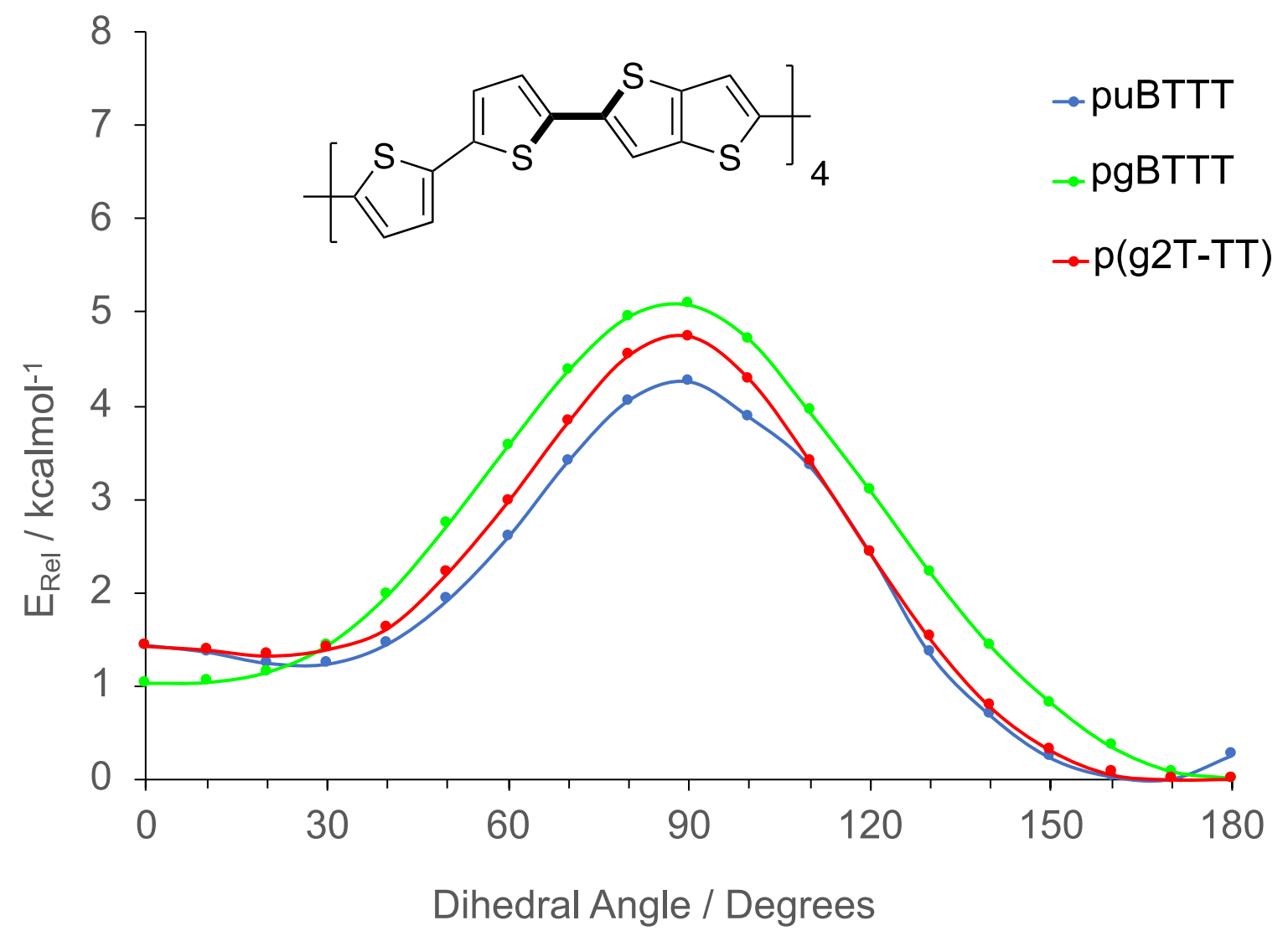

Figure S5. Torsional potential energy surface for puBTTT (blue), pgBTTT (green) and pg2T-TT (red) polymers about the centermost T-TT bond of model oligomers with 4 repeat units, calculated using B3LYPD3/6-31G. The rotated bond is highlighted as bold, and the $180^{\circ}$ conformer is shown. 


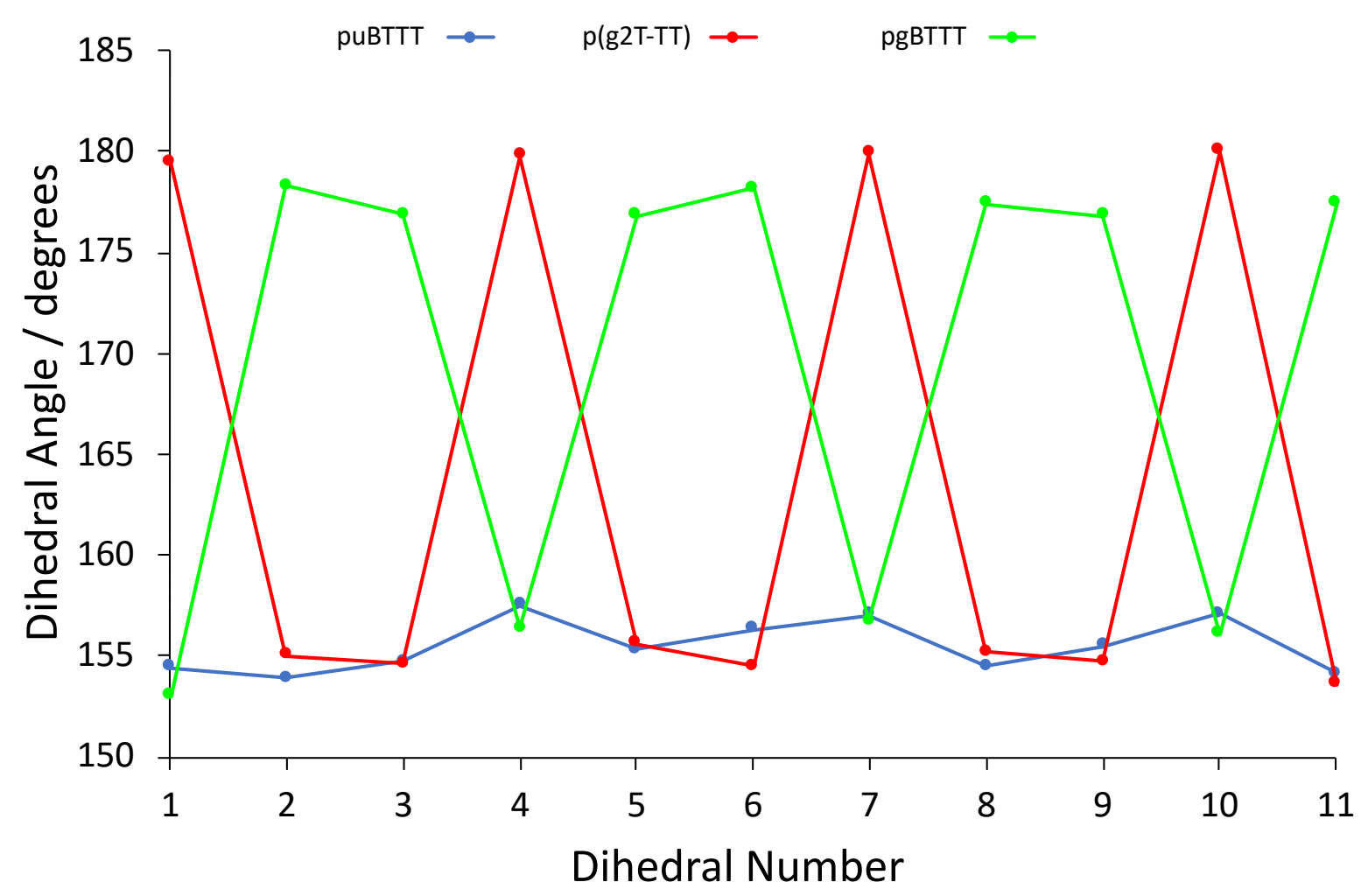

Figure S6. Variation in dihedral angle along the polymer for puBTTT (blue), pgBTTT (green) and p(g2TTT) (red), with pgBTTT exhibiting the largest number of near planar dihedral angles. Geometries of oligomers of 4 repeat units were optimized with $\omega \mathrm{B} 97 \mathrm{XD} / 6-31 \mathrm{G}^{*}$ with $\omega=0.1 \mathrm{Bohr}^{-1}$.
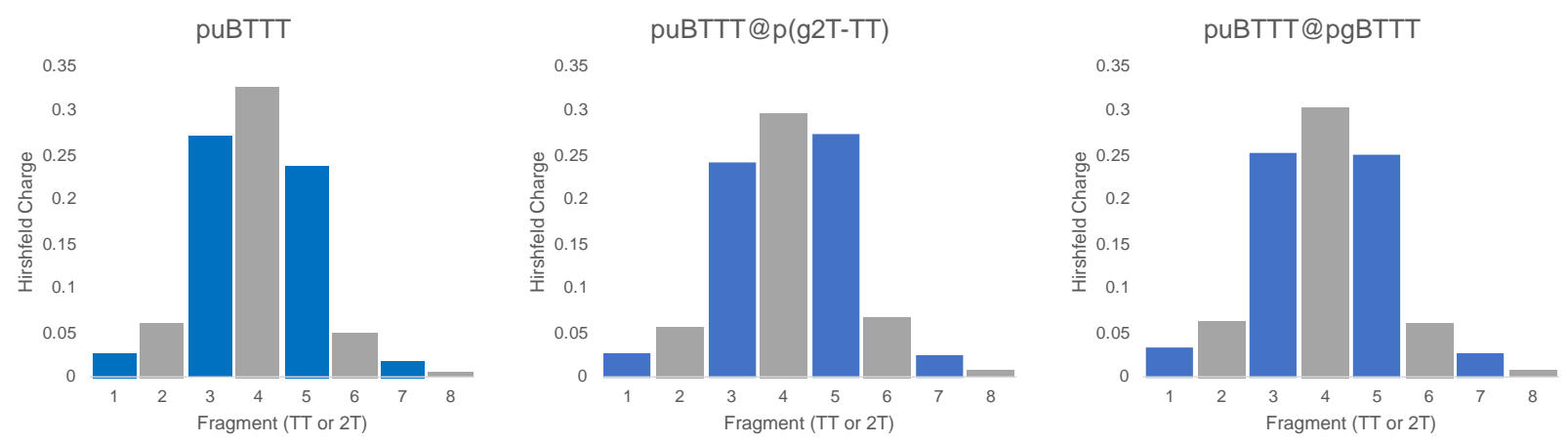

Figure S7. Hirshfeld charge distribution in oxidized puBTTT, puBTTT with dihedrals fixed at the $\mathrm{p}(\mathrm{g} 2 \mathrm{~T}-$ TT) geometry $(\mathrm{p}(2 \mathrm{~T}-\mathrm{TT}) @ \mathrm{p}(\mathrm{g} 2 \mathrm{~T}-\mathrm{TT}))$ and puBTTT with dihedrals fixed at the pgBTTT geometry $(\mathrm{p}(2 \mathrm{~T}-$ TT)@pgBTTT). Calculated with IP-tuned $\omega$ B97XD/6-31G* in chloroform polarizable continuum. 


\section{Thermal analysis}

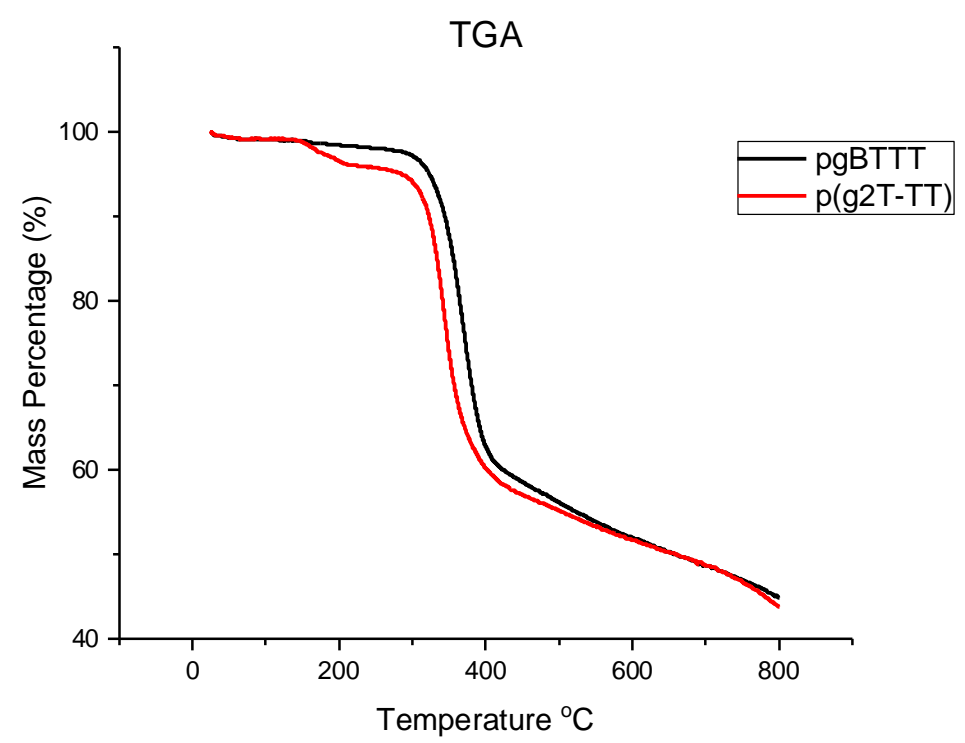

Figure S8. Thermogravimetric analysis (TGA) of pgBTTT and p(g2T-TT) 

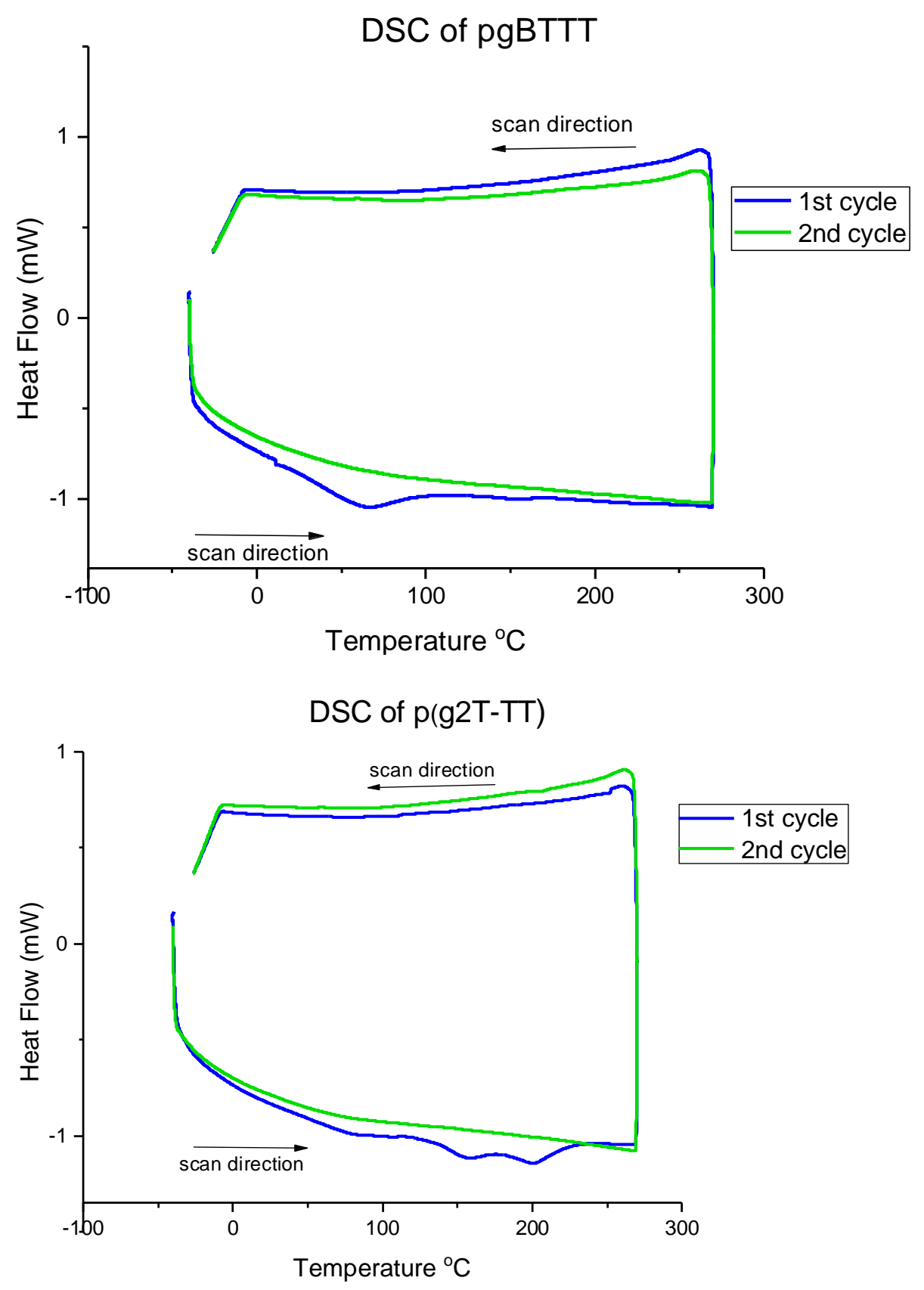

Figure S9. Differential scanning calorimetry (DSC) of pgBTTT and p(g2T-TT) (exo up). 
Differential scanning calorimetry (DSC) was carried out on $\mathrm{p}(\mathrm{g} 2 \mathrm{~T}-\mathrm{TT})$ and pgBTTT affording similar traces (see Figure S9) with no apparent glass transitions. However, pgBTTT exhibits a heating endotherm peak around $65^{\circ} \mathrm{C}$ with no observed peaks during the cooling process. Moreover, no peaks were observed in the second cycle. The same behavior was recorded with $\mathrm{p}(\mathrm{g} 2 \mathrm{~T}-\mathrm{TT})$ with three endotherm peaks at 156 , and $201^{\circ} \mathrm{C}$ found during the heating step, and with no peaks detected in the cooling step or the second heating/cooling cycle.

Thermal gravimetric analysis (TGA) was performed to evaluate the thermal stability of the polymers. Both polymers start to decompose after $300{ }^{\circ} \mathrm{C}$ (see SI), with p(g2T-TT) exhibiting a loss of $2.7 \%$ at $150{ }^{\circ} \mathrm{C}$, followed by a maximum thermal degradation at $320^{\circ} \mathrm{C}$. The (TGA) for pgBTTT was slightly different, showing no weight loss at $150{ }^{\circ} \mathrm{C}$ and a maximum thermal degradation temperature of $340{ }^{\circ} \mathrm{C}$. The improved thermal stability could be an indication of a more ordered structure for pgBTTT, and the absence of weight loss at $150{ }^{\circ} \mathrm{C}$ can tentatively be an indication of a more ordered and packed glycol side chains, making it difficult for pgBTTT to absorb moisture in the absence of a voltage.

\section{Organic electrochemical transistors}

4

a
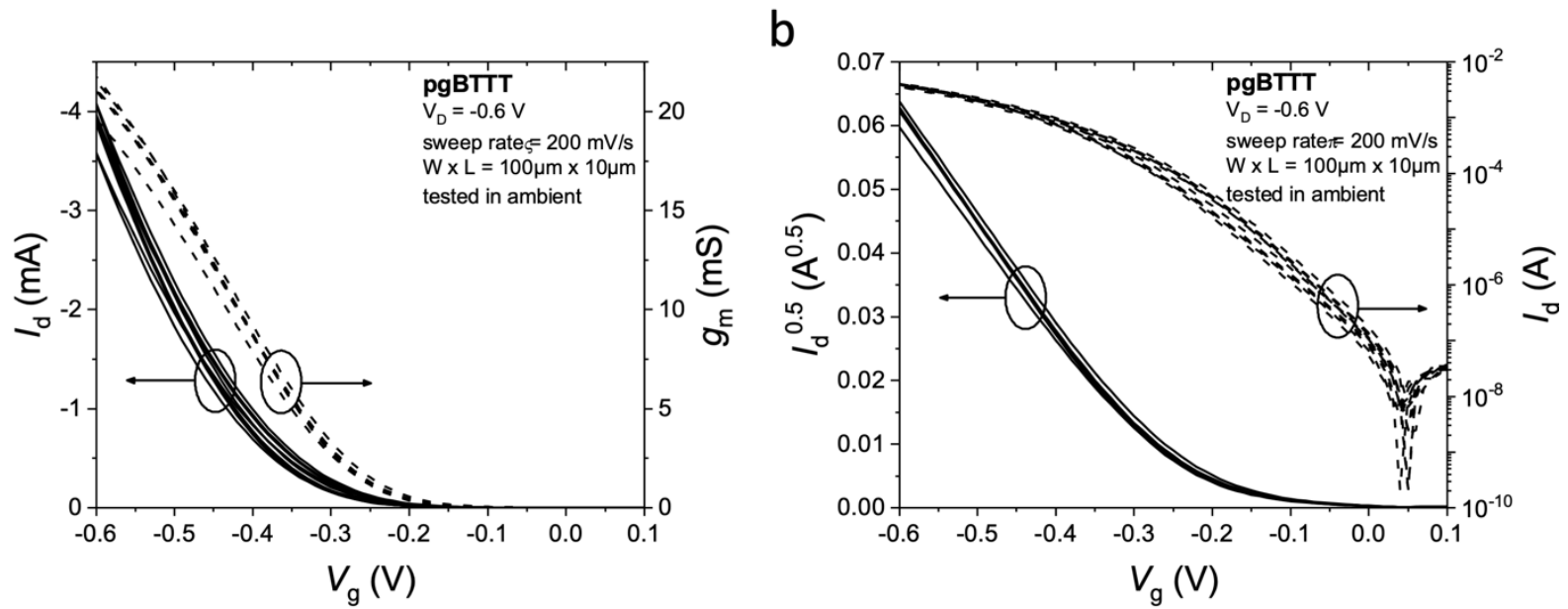

Figure S10. pgBTTT OECT Device-to-Device Variability: (a) Transfer curves $\left(\mathrm{I}_{\mathrm{d}} \mathrm{vs}_{\mathrm{g}} \mathrm{V}_{\mathrm{g}}\right.$ ) and voltage dependent transconductance $\left(g_{m}\right)$ of six pgBTTT devices, along with (b) $I_{d}{ }^{1 / 2}$ vs $V_{g}$ plots and semi-log transfer curves for the same six devices used to extract saturation mobility and subthreshold swing, respectively. 


\section{EIS measurements}
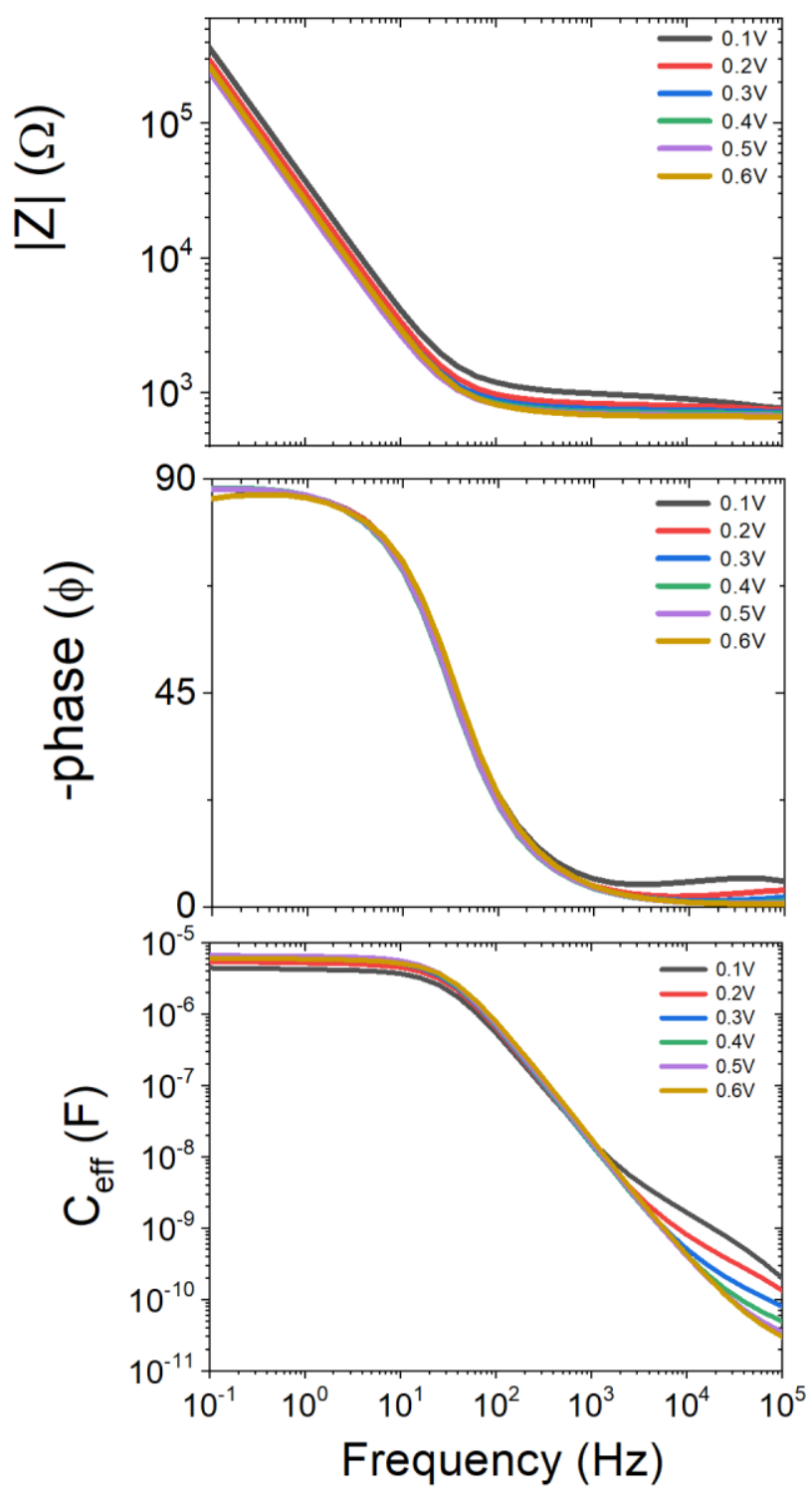

Figure S11. Electrochemical Impedance Spectroscopy: Representative frequency dependent (a) impedance, (b) phase, and (c) effective capacitance of pgBTTT thin films, used to calculate volumetric capacitance $\left(C^{*}\right)$. 


\section{GIWAXS}

Table S1. GIWAXS fit peak coherence lengths estimated from the Scherrer equation.

\begin{tabular}{lcccccc} 
& \multicolumn{2}{c}{ In-plane } & & \multicolumn{2}{c}{ Out-of-plane } \\
\cline { 3 - 4 } Index & & pgBTTT & P(g2T-TT $)$ & & pgBTTT & P(g2T-TT) \\
\cline { 3 - 4 } & & & 39.5 & & 25.1 & 19.1
\end{tabular}

a

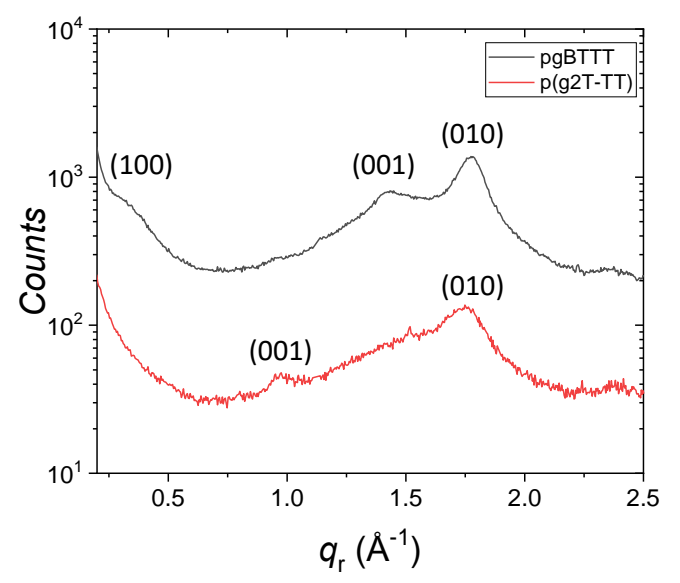

b

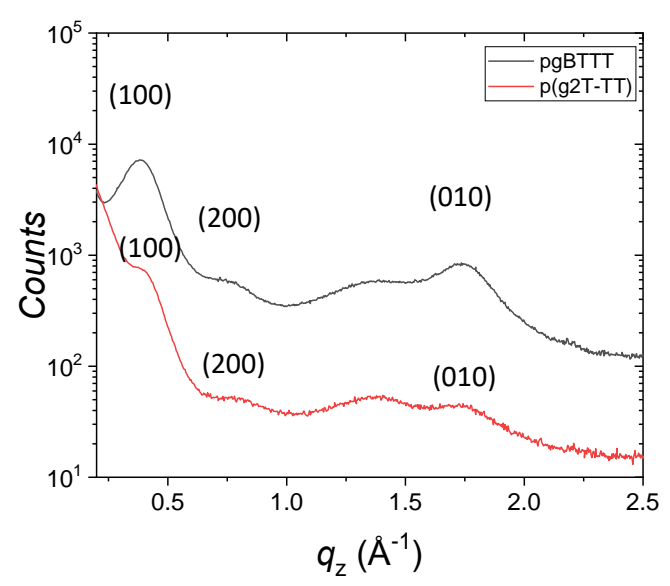

Figure S12. (a) In-plane and (b) out of plane line cuts from two-dimensional grazing incidence X-ray qr-qz scattering map of pgBTTT (black line) and p(g2T-TT) (red line) thin films. 


\section{Scanning Tunneling Microscopy}
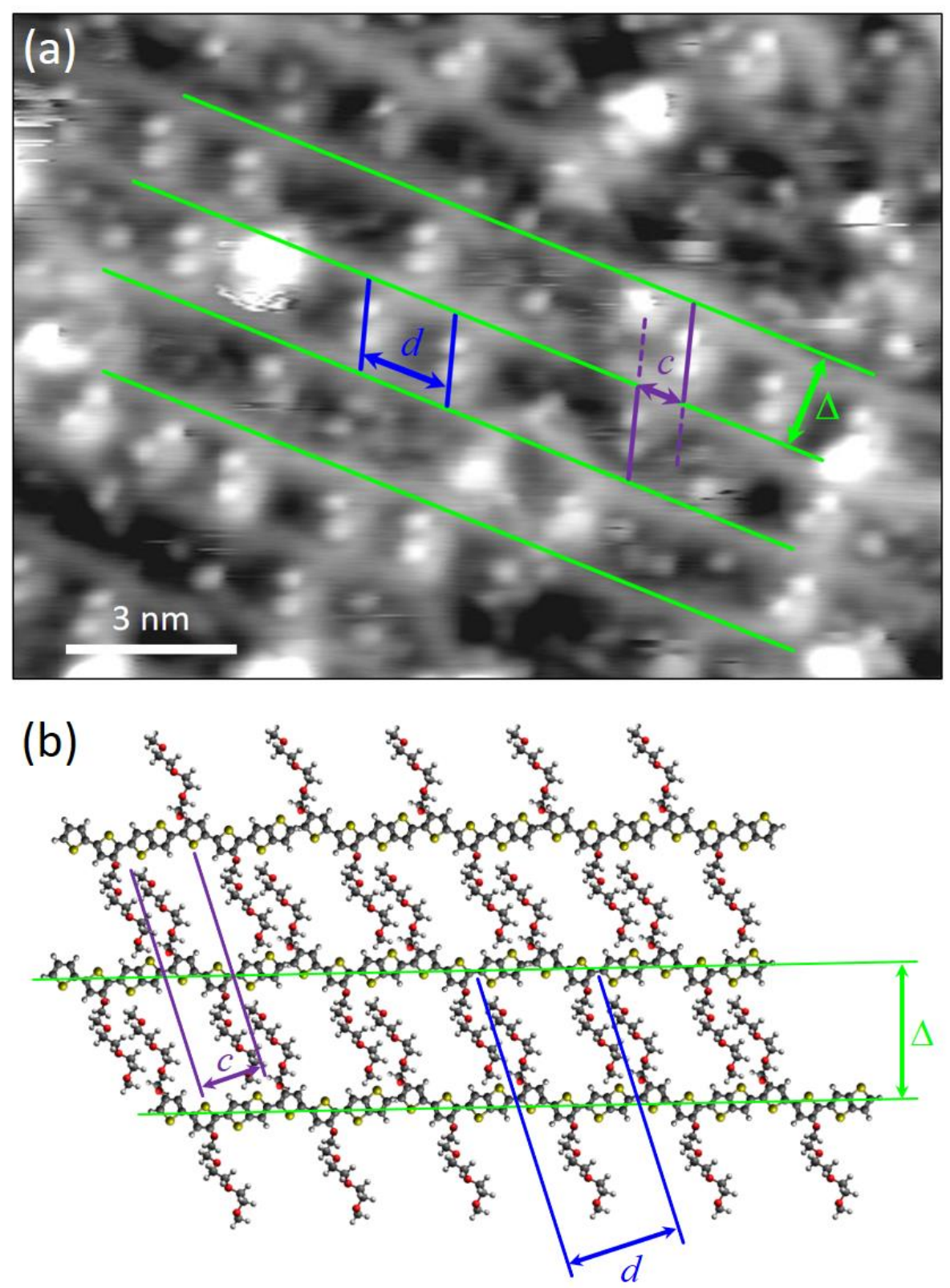

Figure S13. (a) Small scale STM image shown in Fig. 6d of the main text, with a schematic representation of the structural parameters determined experimentally for the 2D assembly of ESD-deposited polymers on $\mathrm{Au}(111)$. (b) The same structural parameters identified in the geometry-optimized molecular model for pgBTTT. 
The green lines in Figure S13a run along the locally parallel backbones of pgBTTT and their separation, $\Delta$, is the $2 \mathrm{D}$ equivalent of the lamellar spacing (see also Figure S13b). The spacing $d$ (blue) represents the distance between successive pairs of double dots on the same side of the backbone and corresponds to the polymer repeat unit length. The repeat unit length of pgBTTT was calculated as the average of a 4-oligomer (with proton substituted side chains and optimized with the MMFF94s force field in the Avogadro molecular editor), resulting in a value $d=13.75 \AA$. Finally, the spacing $c$ (purple) represents the separation between pairs of successive double dots on opposite sides of the backbone, as measured along the local orientation of the backbone. In the model, this corresponds to the distance between the 2- and 4- positions of two successive thiophenes, equivalent to $c=6.42 \AA$.

pgBTTT
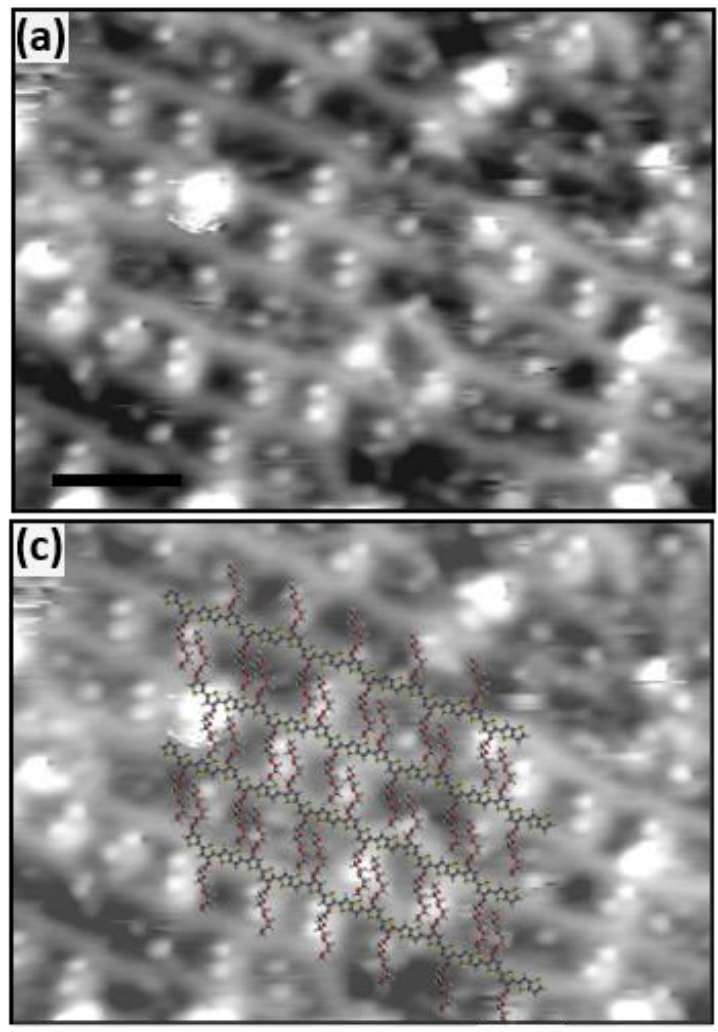

$\mathrm{p}(\mathrm{g} 2 \mathrm{~T}-\mathrm{TT})$
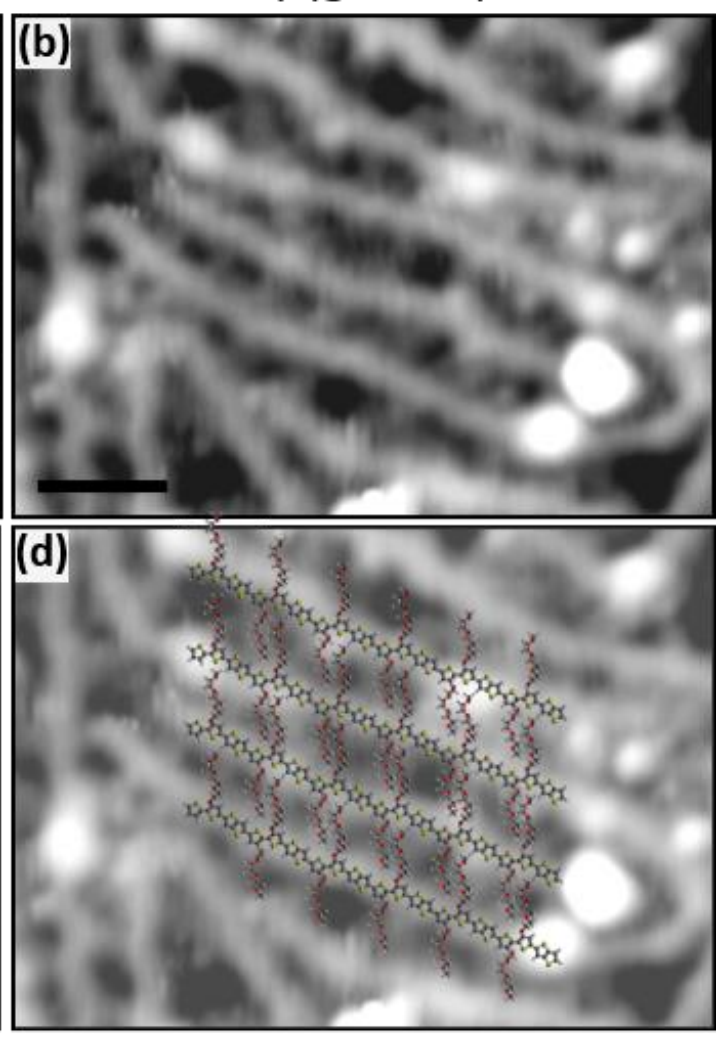

Figure S14. Small scale STM images for pgBTTT (a) and p(g2T-TT) (b), as reported in the main text (Fig. $6 \mathrm{c}$ and $6 \mathrm{~d}$ ). The geometry-optimized models showed in Figs. $6 \mathrm{e}$ and $6 \mathrm{f}$ have been superposed for the two polymers in panels (c) and (d), respectively. A good agreement with the STM images is visible for the backbones and for the interdigitating triethylene glycol side chains. 


\section{Spectroelectrochemistry}
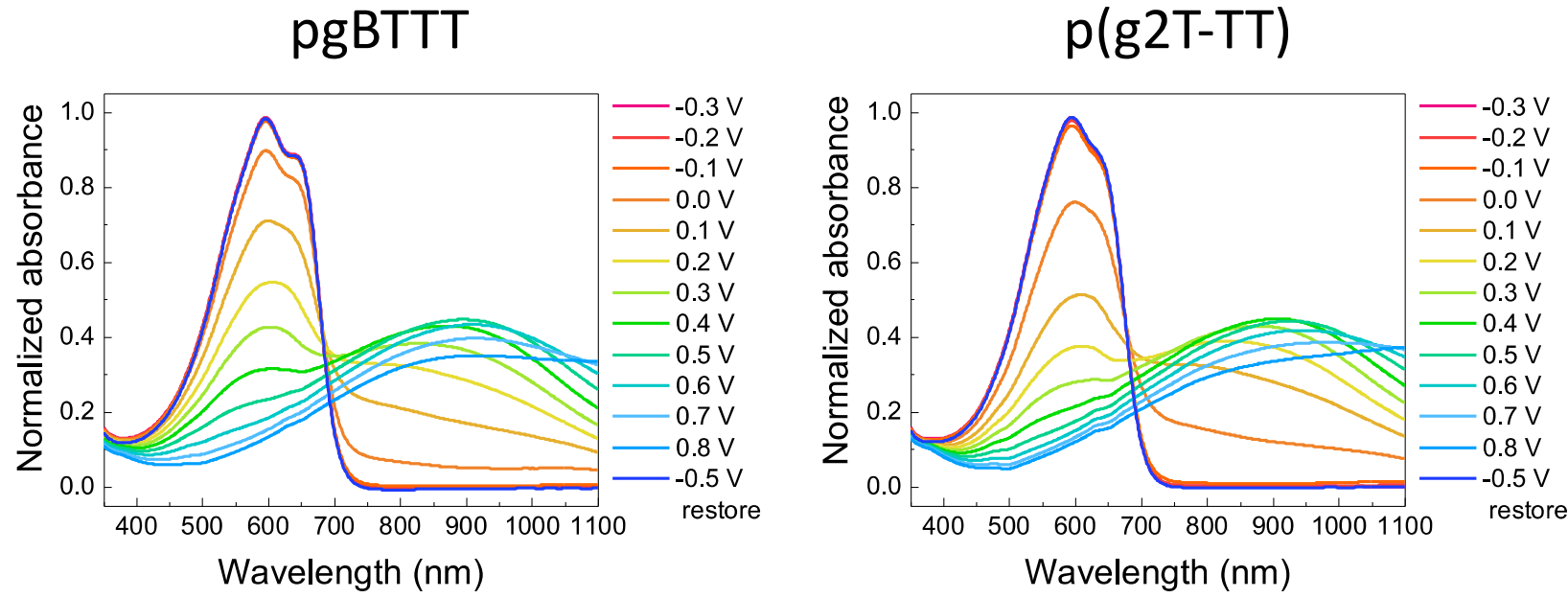

Figure S15. Spectroelectrochemistry in acetonitrile $\left(0.1 \mathrm{M} \mathrm{TBA} \mathrm{PF}_{6}\right)$ of pgBTTT and pg2T-TT, films deposited on ITO. Spectra are normalized to the peak absorption of the neutral spectra.
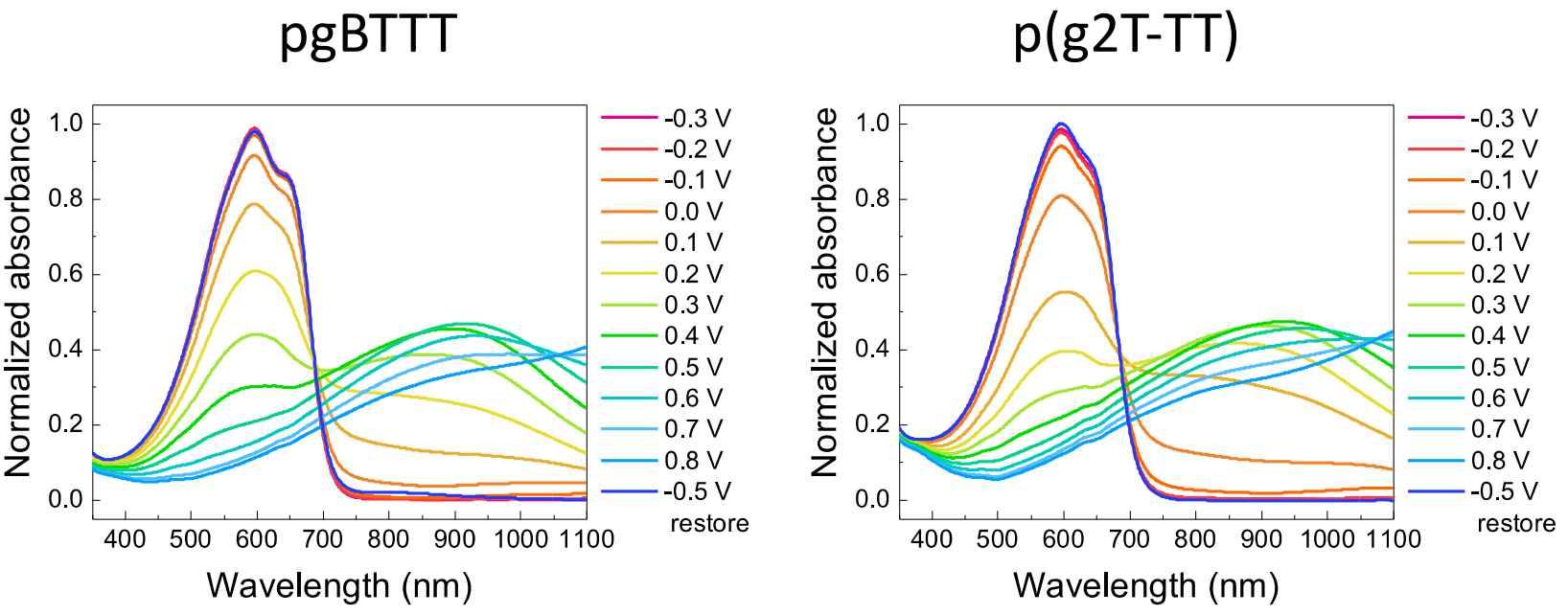

Figure S16. Spectroelectrochemistry in water $(0.1 \mathrm{M} \mathrm{NaCl})$ of pgBTTT and pg2T-TT, films deposited on ITO. Spectra are normalized to the peak absorption of the neutral spectra 


\section{Cyclic Voltammetry}
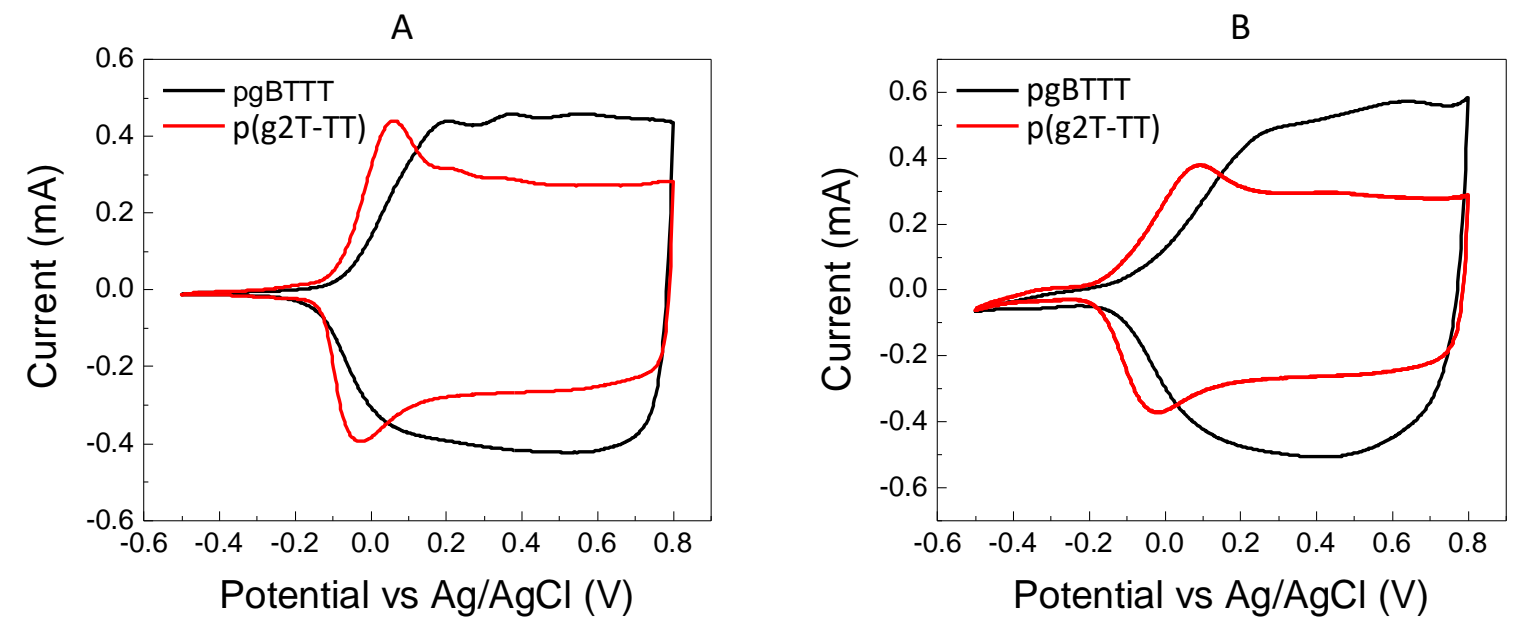

Figure S17. Cyclic voltammetry measurements of pgBTTT, black, and pg2T-TT, red, carried out in anhydrous degassed 0.1 M TBAPF6 acetonitrile solution (A.) and in aqueous $0.1 \mathrm{M} \mathrm{NaCl}$ solution (B.) with scan rates of $100 \mathrm{mV} / \mathrm{s}$.

\section{EQCM-D measurements}
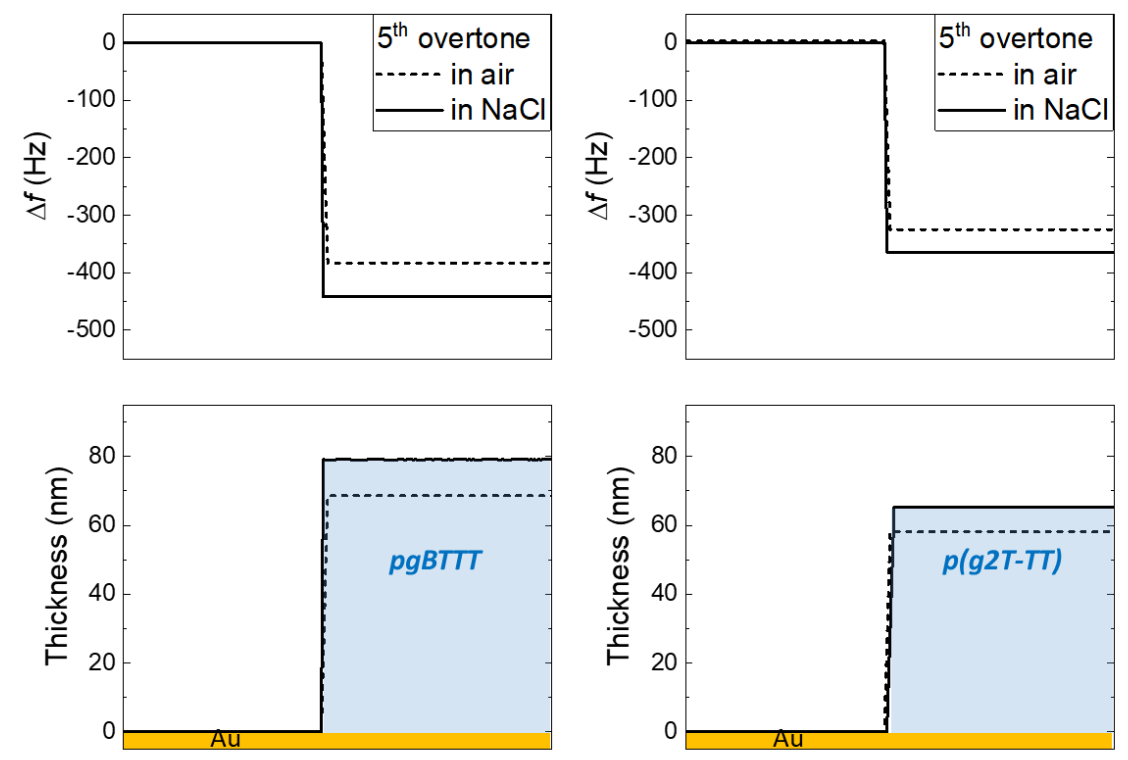

Figure S18. Frequency shifts $(\Delta f)$ and the corresponding thicknesses of the polymer films cast on Au coated sensors, recorded in air and when immersed in $\mathrm{NaCl}_{(\mathrm{aq} .)} 0.1 \mathrm{M}$. Here, we show the $\Delta f$ for the $5^{\text {th }}$ overtone. 


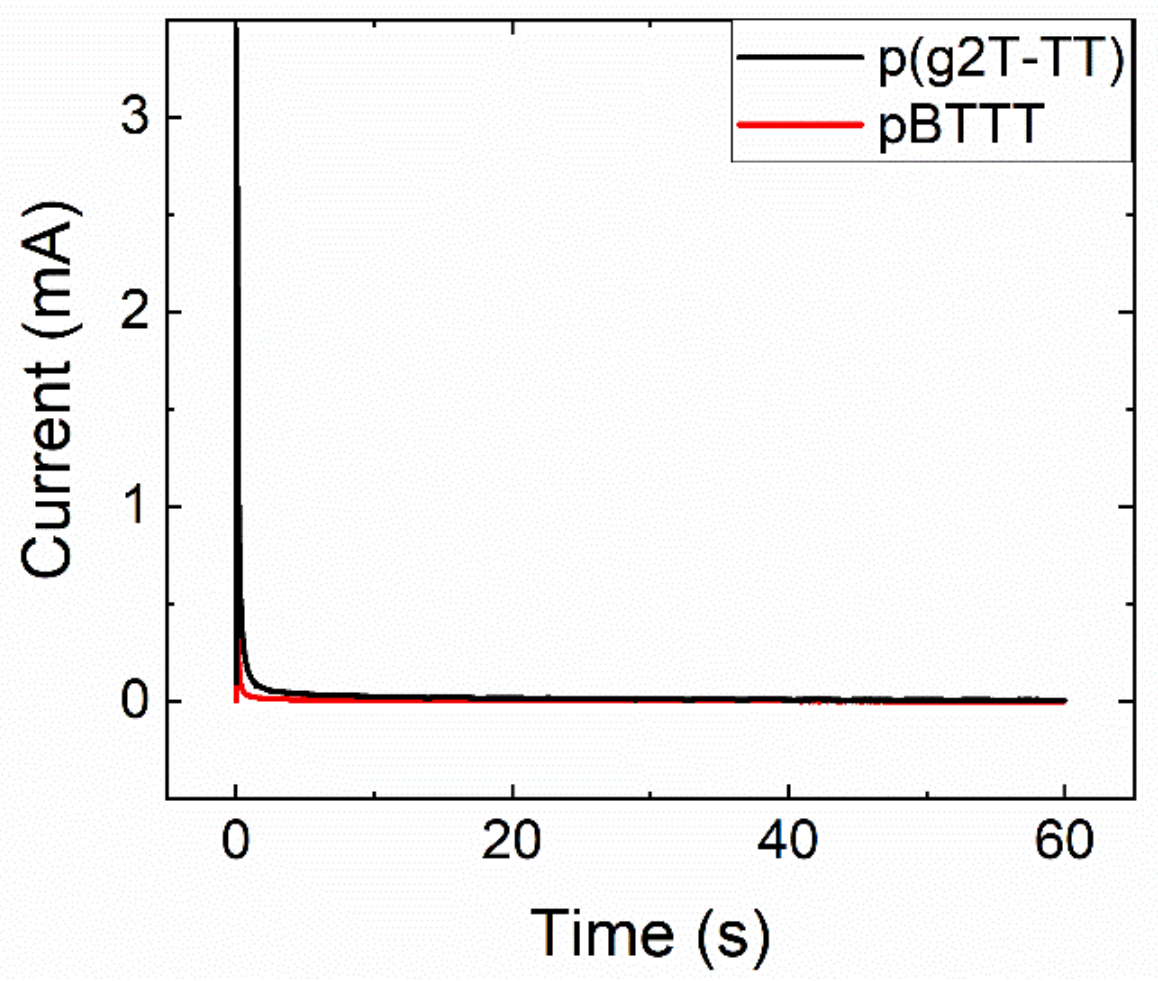

Figure S19. Current vs. time profiles for $\mathrm{p}(\mathrm{g} 2 \mathrm{~T}-\mathrm{TT})$ (black line) and for pgBTTT (red line) and recorded during the eQCM-D measurements. 


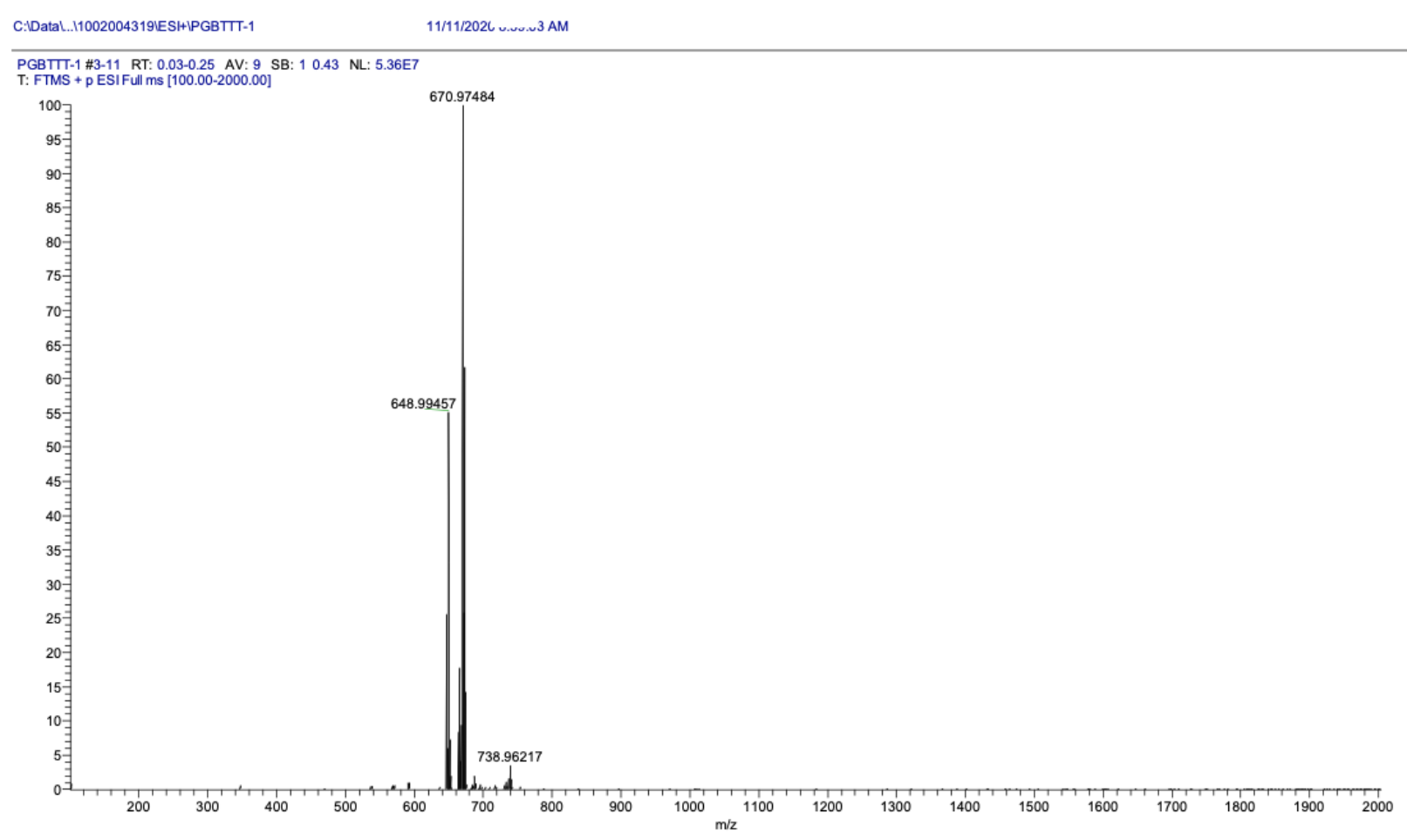

Figure S20. ESI+MS full scan of compound 4

\section{References}

1. Frisch, M. J.; Trucks, G. W.; Schlegel, H. B.; Scuseria, G. E.; Robb, M. A.; Cheeseman, J. R.; Scalmani, G.; Barone, V.; Petersson, G. A.; Nakatsuji, H.; Li, X.; Caricato, M.; Marenich, A. V.; Bloino, J.; Janesko, B. G.; Gomperts, R.; Mennucci, B.; Hratchian, H. P.; Ortiz, J. V.; Izmaylov, A. F.; Sonnenberg, J. L.; Williams; Ding, F.; Lipparini, F.; Egidi, F.; Goings, J.; Peng, B.; Petrone, A.; Henderson, T.; Ranasinghe, D.; Zakrzewski, V. G.; Gao, J.; Rega, N.; Zheng, G.; Liang, W.; Hada, M.; Ehara, M.; Toyota, K.; Fukuda, R.; Hasegawa, J.; Ishida, M.; Nakajima, T.; Honda, Y.; Kitao, O.; Nakai, H.; Vreven, T.; Throssell, K.; Montgomery Jr., J. A.; Peralta, J. E.; Ogliaro, F.; Bearpark, M. J.; Heyd, J. J.; Brothers, E. N.; Kudin, K. N.; Staroverov, V. N.; Keith, T. A.; Kobayashi, R.; Normand, J.; Raghavachari, K.; Rendell, A. P.; Burant, J. C.; Iyengar, S. S.; Tomasi, J.; Cossi, M.; Millam, J. M.; Klene, M.; Adamo, C.; Cammi, R.; Ochterski, J. W.; Martin, R. L.; Morokuma, K.; Farkas, O.; Foresman, J. B.; Fox, D. J. Gaussian 16 Rev. C.01, Wallingford, CT, 2016.

2. Körzdörfer, T.; Brédas, J.-L., Organic Electronic Materials: Recent Advances in the DFT Description of the Ground and Excited States Using Tuned Range-Separated Hybrid Functionals. Accounts of Chemical Research 2014, 47 (11), 3284-3291.

3. Jiang, Z., GIXSGUI: a MATLAB toolbox for grazing-incidence X-ray scattering data visualization and reduction, and indexing of buried three-dimensional periodic nanostructured films. Journal of Applied Crystallography 2015, 48 (3), 917-926.

4. Horcas, I.; Fernández, R.; Gómez-Rodríguez, J. M.; Colchero, J.; Gómez-Herrero, J.; Baro, A. M., WSXM: A software for scanning probe microscopy and a tool for nanotechnology. Review of Scientific Instruments 2007, 78 (1), 013705. 
5. Hanwell, M. D.; Curtis, D. E.; Lonie, D. C.; Vandermeersch, T.; Zurek, E.; Hutchison, G. R., Avogadro: an advanced semantic chemical editor, visualization, and analysis platform. Journal of Cheminformatics 2012, 4 (1), 17.

6. LMAPper - The SPM and Mol Viewer. https://sourceforge.net/projects/spm-and-mol-viewer/.

7. Parr, Z. S.; Rashid, R. B.; Paulsen, B. D.; Poggi, B.; Tan, E.; Freeley, M.; Palma, M.; Abrahams, I.; Rivnay, J.; Nielsen, C. B., Semiconducting Small Molecules as Active Materials for $\mathrm{p}$-Type Accumulation Mode Organic Electrochemical Transistors. Advanced Electronic Materials 2020, 6 (6), 2000215.

8. Tarabella, G.; Santato, C.; Yang, S. Y.; lannotta, S.; Malliaras, G. G.; Cicoira, F., Effect of the gate electrode on the response of organic electrochemical transistors. Applied Physics Letters 2010, 97 (12), 123304.

9. Savva, A.; Cendra, C.; Giugni, A.; Torre, B.; Surgailis, J.; Ohayon, D.; Giovannitti, A.; McCulloch, I.; Di Fabrizio, E.; Salleo, A.; Rivnay, J.; Inal, S., Influence of Water on the Performance of Organic Electrochemical Transistors. Chemistry of Materials 2019, 31 (3), 927-937.

10. Savva, A.; Hallani, R.; Cendra, C.; Surgailis, J.; Hidalgo, T. C.; Wustoni, S.; Sheelamanthula, R.; Chen, X.; Kirkus, M.; Giovannitti, A.; Salleo, A.; McCulloch, I.; Inal, S., Balancing lonic and Electronic Conduction for High-Performance Organic Electrochemical Transistors. Advanced Functional Materials 2020, 30 (11), 1907657.

11. Gunathilake, S. S.; Magurudeniya, H. D.; Huang, P.; Nguyen, H.; Rainbolt, E. A.; Stefan, M. C.; Biewer, M. C., Synthesis and characterization of novel semiconducting polymers containing pyrimidine. Polymer Chemistry 2013, 4 (20), 5216-5219. 